Supporting Information for

\title{
2-Ene-1,4-diols by Dimerization of Terminal Epoxides using Hindered Lithium Amides
}

\author{
David M. Hodgson, ${ }^{\dagger}{ }^{\dagger}$ Christopher D. Bray ${ }^{\dagger}$ and Nicholas D. Kindon ${ }^{\ddagger}$ \\ ${ }^{\dagger}$ Department of Chemistry, University of Oxford, Chemistry Research Laboratory, Mansfield Road, Oxford OX1 3TA, U.K. \\ ${ }^{\ddagger}$ AstraZeneca R\&D Charnwood Medicinal Chemistry, Bakewell Road, Loughborough, Leicestershire LE11 5RH, U.K.
}

\section{Contents}

General Details $\quad$ S2

Spectral Data

(3S,4E,6S)-2,2,7,7-Tetramethyloct-4-ene-3,6-diol $(S, S)-2 \mathbf{a} \quad$ S2

$(1 R, 2 Z, 4 R)-1,4-D i c y c l o h e x y l b u t-2-e n e-1,4-d i o l ~ 3 b \quad S 2$

$(1 R, 2 E, 4 R)-1,4-D i c y c l o h e x y l b u t-2-e n e-1,4-d i o l ~ 3 a \quad S 2$

(1S,4S)-1,4-Dicyclohexylbutane-1,4-diol $7 \quad$ S3

(6R,7Z,9R)-Tetradec-7-ene-6,9-diol 4b S3

(6R,7E,9R)-Tetradec-7-ene-6,9-diol 4a $\quad$ S3

(2S,3E,5S)-1,6-Bis(trityloxy)hex-3-ene-2,5-diol $6 \quad$ S4

${ }^{1} \mathrm{H}$ and ${ }^{13} \mathrm{C}$ NMR Spectra

(3S,4E,6S)-2,2,7,7-Tetramethyloct-4-ene-3,6-diol (S,S)-2a $\quad$ S5

(1R,2E,4R)-1,4-Dicyclohexylbut-2-ene-1,4-diol 3a $\quad$ S6

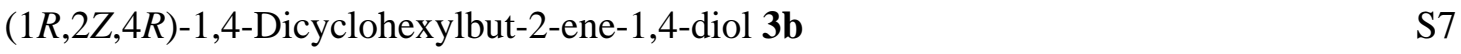

(1S,4S)-1,4-Dicyclohexylbutane-1,4-diol $7 \quad$ S8

$(6 R, 7 E, 9 R)$-Tetradec-7-ene-6,9-diol 4a $\quad$ S9

(6R,7Z,9R)-Tetradec-7-ene-6,9-diol 4b $\quad$ S10

(2S,3E,5S)-1,6-Bis(trityloxy)hex-3-ene-2,5-diol $6 \quad$ S11

Synthetic D-mannitol $\quad$ S12

Natural D-mannitol $\quad$ S13

Synthetic D-iditol $\quad$ S14

$\begin{array}{ll}\text { Natural L-iditol } & \text { S15 }\end{array}$

X-ray Data

(3S,4E,6S)-2,2,7,7-Tetramethyloct-4-ene-3,6-diol (S,S)-2a $\quad$ S16

$\left(3 R^{*}, 4 Z, 6 R^{*}\right)$-2,2,7,7-Tetramethyloct-4-ene-3,6-diol 2b $\quad$ S16

$(1 R, 2 E, 4 R)-1,4-D i c y c l o h e x y l b u t-2-e n e-1,4-d i o l ~ 3 a \quad S 16$

$\begin{array}{ll}\text { References } & \text { S17 }\end{array}$ 


\section{General Details}

All reactions required anhydrous conditions and were conducted in flame-dried apparatus under an atmosphere of argon. THF was filtered through activated alumina; TMP and $t$ BuOMe were distilled from $\mathrm{CaH}_{2}$; all other reagents were used as received. Column chromatography was carried out on Kieselgel $60(40-63 \mu \mathrm{m})$ silica gel unless otherwise stated. Light petrol refers to the fraction of petroleum boiling between $30{ }^{\circ} \mathrm{C}$ and $40{ }^{\circ} \mathrm{C}$. IR spectra were recorded as thin films or $\mathrm{KBr}$ discs, using a 1750 FTIR spectrophotometer. ${ }^{1} \mathrm{H}$ and ${ }^{13} \mathrm{C}$ NMR spectra were recorded at $25{ }^{\circ} \mathrm{C}$, with DPX250, DPX400, AV400 or AMX500 spectrometers unless otherwise stated. Chemical shifts are reported relative to $\mathrm{CHCl}_{3}\left[{ }^{1} \mathrm{H} \delta\right.$ 7.27]/CDCl ${ }_{3}\left[{ }^{13} \mathrm{C} \delta 77.0\right]$ or $\mathrm{MeOH}-\mathrm{d}_{4}\left[{ }^{1} \mathrm{H} \delta 3.31,{ }^{13} \mathrm{C} \delta 49.0\right]$. Coupling constants $(J)$ are given in $\mathrm{Hz}$, multiplicities are given as multiplet (m), singlet (s) (and where applicable are described as broad (b)), doublet (d) or triplet (t). Mass spectra were obtained using a GCT spectrometer or by the EPSRC National Mass Spectrometry Service Centre at the University of Swansea using a high resolution double focusing mass spectrometer with tandem ion trap.

\section{Spectral Data}

(3S,4E,6S)-2,2,7,7-Tetramethyloct-4-ene-3,6-diol (S,S)-2a ${ }^{1}$

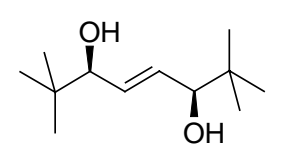

According to the general procedure $(R)$-tert-butyloxirane $(244 \mu \mathrm{L}, 2.00$ mmol) gave enediol $(S, S)-2 a$ as a white solid (172 mg, 86\%); $R_{\mathrm{f}} 0.41$ (90\% $\mathrm{Et}_{2} \mathrm{O}$ in petrol); mp 119-120 ${ }^{\circ} \mathrm{C}$ (lit. $\left.{ }^{1} 120-121^{\circ} \mathrm{C}\right) ;[\alpha]_{\mathrm{D}}{ }^{24}-52.7(c=$ 1.0, MeOH) (lit. $\left.{ }^{1}-55.4(c=2.17, \mathrm{MeOH})\right) ; \mathrm{IR}_{\max } / \mathrm{cm}^{-1}(\mathrm{KBr}) 3419,2964,1473,1363$, 1313, 1265, 1085, 1051, 1003; ${ }^{1} \mathrm{H}$ NMR (400 MHz, MeOH-d $) \delta$ 5.72-5.63 (m, 2H, $2 \times$ $=\mathrm{CH}), 4.91$ (bs, $2 \mathrm{H}, 2 \times \mathrm{OH}), 3.73-3.65(\mathrm{~m}, 2 \mathrm{H}, 2 \times \mathrm{OCH}), 0.91\left(\mathrm{~s}, 18 \mathrm{H}, 2 \times \mathrm{CMe}_{3}\right) ;{ }^{13} \mathrm{C}$ NMR (100 MHz, MeOH-d 4$) \delta 133.3(2 \times=\mathrm{CH}), 81.6(2 \times \mathrm{OCH}), 35.9\left(2 \times \mathrm{CMe}_{3}\right), 26.4(2 \times$ $\mathrm{CMe}_{3}$ ); Anal. calcd for $\mathrm{C}_{12} \mathrm{H}_{24} \mathrm{O}_{2}$ : C, 71.95; H, 12.08. Found C, 71.87; H, 11.98 .

$(1 R, 2 Z, 4 R)-1,4-D i c y c l o h e x y l b u t-2-e n e-1,4-d i o l \quad 3 b$ and $(1 R, 2 E, 4 R)-1,4-d i c y c l o h e x y l b u t-2-$ ene-1,4-diol $3 \mathbf{a}^{1}$

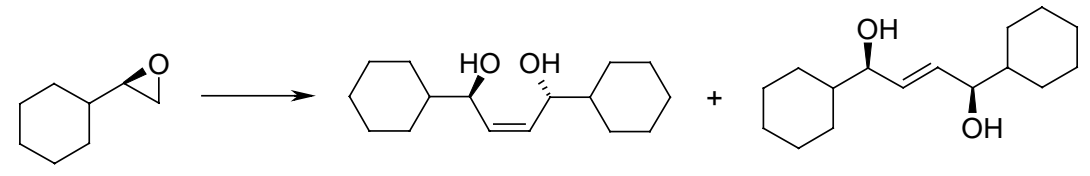

According to the general procedure $(R)$-cyclohexyloxirane $(271 \mu \mathrm{L}, 2.00 \mathrm{mmol})$ first of all gave (Z)-enediol $3 \mathbf{b}$ as a white solid (66 mg, 26\%); $R_{\mathrm{f}} 0.70\left(\mathrm{Et}_{2} \mathrm{O}\right) ; \mathrm{mp} 110-11{ }^{\circ} \mathrm{C}$; $[\alpha]_{\mathrm{D}}{ }^{24}$ $-2.4\left(c=0.25, \mathrm{CHCl}_{3}\right)$; IR $\mathrm{v}_{\max } / \mathrm{cm}^{-1}(\mathrm{KBr}) 3383,2922,2851,1449,1083,1010 ;{ }^{1} \mathrm{H} \mathrm{NMR}$ (400 MHz, MeOH-d $\left.{ }_{4}\right) \delta$ 5.47-5.41 (m, 2H, $\left.2 \times=\mathrm{CH}\right), 4.90$ (bs, 2H, $\left.2 \times \mathrm{OH}\right), 4.09-4.01(\mathrm{~m}$, 
$2 \mathrm{H}, 2 \times \mathrm{OCH}), 2.02-0.80(\mathrm{~m}, 22 \mathrm{H}, 2 \times \mathrm{Cy}) ;{ }^{13} \mathrm{C}$ NMR $\left(100 \mathrm{MHz} \mathrm{MeOH}-\mathrm{d}_{4}\right) \delta 133.8(2 \times$ $=\mathrm{CH}), 73.1(2 \times \mathrm{OCH}), 45.6(2 \times \mathrm{CH}), 29.9\left(2 \times \mathrm{CH}_{2}\right), 29.9\left(2 \times \mathrm{CH}_{2}\right), 27.8\left(2 \times \mathrm{CH}_{2}\right), 27.4$ $\left(2 \times \mathrm{CH}_{2}\right), 27.3\left(2 \times \mathrm{CH}_{2}\right)$; HRMS $\mathrm{m} / \mathrm{z}\left(\mathrm{M}+\mathrm{NH}_{4}{ }^{+}\right)$Found: 270.2430. $\mathrm{C}_{16} \mathrm{H}_{32} \mathrm{O}_{2} \mathrm{~N}$ requires 270.2428. Second to elute was (E)-enedio1 3a as a white solid (129 mg, 51\%); $R_{\mathrm{f}} 0.30$ $\left(\mathrm{Et}_{2} \mathrm{O}\right)$; mp $114-116{ }^{\circ} \mathrm{C}\left(\right.$ lit. $\left.^{1} 117-120{ }^{\circ} \mathrm{C}\right) ;[\alpha]_{\mathrm{D}}{ }^{24}-30.3$ (c=1.0, $\left.\mathrm{CHCl}_{3}\right)\left(\right.$ lit. $^{1}+39.0(c=1.5$, $\mathrm{CHCl}_{3}$ ) for (ent)-3a (82\% ee); IR v $\mathrm{max}_{\max } / \mathrm{cm}^{-1}(\mathrm{KBr}) 3411,2919,2838,2441,1618,1448,1011$; ${ }^{1} \mathrm{H}$ NMR (400 MHz, MeOH-d ${ }_{4}$ ) $\delta .60-5.57$ (m, 2H, $2 \times=\mathrm{CH}$ ), 4.91 (bs, $2 \mathrm{H}, 2 \times \mathrm{OH}$ ), 3.80$3.72(\mathrm{~m}, 2 \mathrm{H}, 2 \times \mathrm{OCH}), 2.00-0.80$ (m, 22H, $2 \times \mathrm{Cy}) ;{ }^{13} \mathrm{C} \mathrm{NMR}\left(\mathrm{MeOH}-\mathrm{d}_{4}, 100 \mathrm{MHz}\right) \delta 134.1$ $(2 \times=\mathrm{CH}), 78.0(2 \times \mathrm{OCH}), 45.2(2 \times \mathrm{CH}), 30.1\left(4 \times \mathrm{CH}_{2}\right), 27.8\left(2 \times \mathrm{CH}_{2}\right), 27.4\left(2 \times \mathrm{CH}_{2}\right)$, $27.3\left(2 \times \mathrm{CH}_{2}\right)$.

$(1 S, 4 S)-1,4-D i c y c l o h e x y l b u t a n e-1,4-d i o l ~ 7^{1}$

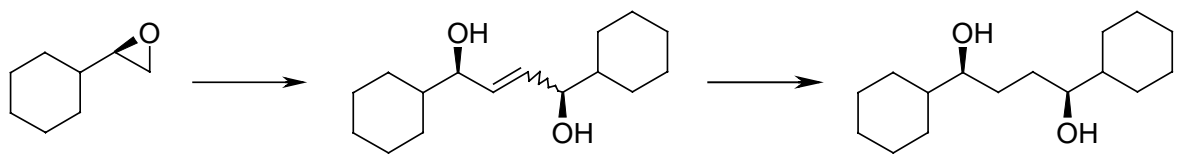

(R)-Cyclohexyloxirane (271 $\mu \mathrm{L}, 2.00 \mathrm{mmol}$ ) was dimerized according to the general procedure, following addition of $\mathrm{MeOH}\left(3 \mathrm{~cm}^{3}\right)$, the reaction was separated between EtOAc $\left(10 \mathrm{~cm}^{3}\right)$ and $0.05 \mathrm{M} \mathrm{H}_{3} \mathrm{PO}_{4}\left(10 \mathrm{~cm}^{3}\right)$. The aqueous phase was extracted with EtOAc $\left(10 \mathrm{~cm}^{3}\right)$ and the combined organic phases were washed with brine $\left(20 \mathrm{~cm}^{3}\right)$ then dried with $\mathrm{MgSO}_{4}$. Removal of the solvent in vacuo gave a colorless oil that was dissolved in EtOH $\left(40 \mathrm{~cm}^{3}\right)$. $5 \% \mathrm{Pt} / \mathrm{C}$ (20 mg) was added and the suspension was stirred under 50 atm of hydrogen for $2 \mathrm{~h}$. Afterwards, the mixture was filtered through a pad of Celite, the solvent was removed in vacuo and the residue was purified by flash chromatography to give diol $\mathbf{7}$ as a white solid (193 mg, 76\%); $R_{\mathrm{f}} 0.31$ (40\% EtOAc in petrol); mp 154-155 ${ }^{\circ} \mathrm{C}$ (lit. ${ }^{1} 116-118{ }^{\circ} \mathrm{C}$ (82\% ee)); $[\alpha]_{\mathrm{D}}{ }^{24}-18.4\left(c=1.0, \mathrm{CHCl}_{3}\right)\left(\right.$ lit. $^{1}-12.5 \quad\left(c=0.6, \mathrm{CHCl}_{3}\right)(82 \%$ ee $)$ ); IR $\mathrm{v}_{\max } / \mathrm{cm}^{-1}(\mathrm{KBr})$ 3398, 2922, 2852; ${ }^{1} \mathrm{H}$ NMR (400 MHz, $\mathrm{CDCl}_{3}$ ) $\delta$ 3.46-3.28 (m, 2H, OCH), 2.11 (bs, 2H, $\mathrm{OH}), 1.90-0.95$ (m, 26H, $12 \times \mathrm{CH}_{2}$ and $\left.2 \times \mathrm{CH}\right) ;{ }^{13} \mathrm{C}$ NMR $\left(100 \mathrm{MHz}, \mathrm{CHCl}_{3}\right) \delta 43.9(2 \times$ $\mathrm{CH}), 30.9\left(2 \times \mathrm{CH}_{2}\right), 29.1\left(2 \times \mathrm{CH}_{2}\right), 27.9\left(2 \times \mathrm{CH}_{2}\right), 26.5\left(2 \times \mathrm{CH}_{2}\right), 26.3\left(2 \times \mathrm{CH}_{2}\right), 26.1(2$ $\left.\times \mathrm{CH}_{2}\right)$.

$(6 R, 7 Z, 9 R)$-Tetradec-7-ene-6,9-diol $\mathbf{4} \mathbf{b}^{2}$ and (6R,7E,9R)-Tetradec-7-ene-6,9-diol $\mathbf{4} \mathbf{a}^{3}$

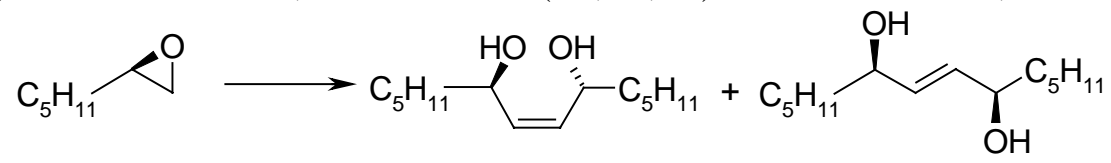

According to the general procedure $(R)-1,2$-epoxyheptane $(271 \mu \mathrm{L}, 2.00 \mathrm{mmol})$ first of all gave (Z)-enediol $4 \mathbf{b}$ as a colorless oil (43 mg, 19\%); $R_{\mathrm{f}} 0.24$ (25\% EtOAc in petrol); $[\alpha]_{\mathrm{D}}{ }^{24} 0$ 
(c = 0.1, $\left.\mathrm{CHCl}_{3}\right) ; \mathrm{IR} \mathrm{v}_{\max } / \mathrm{cm}^{-1}$ (film) 3383, 2930, 2859, 1727, 1466, 1369, 1255, 1175, 1126, 1005; ${ }^{1} \mathrm{H}$ NMR (400 MHz, $\mathrm{CDCl}_{3}$ ) 5.55-5.45 (m, 2H, $\left.2 \times=\mathrm{CH}\right), 4.49-4.37$ (m, 2H, $2 \times$ $\mathrm{OCH}), 1.79-1.23\left(\mathrm{~m}, 18 \mathrm{H}, 8 \times \mathrm{CH}_{2}\right.$ and $\left.2 \times \mathrm{OH}\right), 0.90(\mathrm{t}, 6 \mathrm{H}, J=7,2 \times \mathrm{Me}) ;{ }^{13} \mathrm{C}$ NMR (100 MHz) $134.3(2 \times=\mathrm{CH}), 68.3(2 \times \mathrm{OCH}), 37.6\left(2 \times \mathrm{CH}_{2}\right), 31.7\left(2 \times \mathrm{CH}_{2}\right), 25.1\left(2 \times \mathrm{CH}_{2}\right)$, $22.6\left(2 \times \mathrm{CH}_{2}\right), 14.0(2 \times \mathrm{Me})$. Second to elute was $(E)$-enediol 4a as a colorless oil (99 mg, $43 \%) ; R_{\mathrm{f}} 0.15$ (25\% EtOAc in petrol); $[\alpha]_{\mathrm{D}}{ }^{24}-6.6\left(c=1.0, \mathrm{CHCl}_{3}\right)\left(\right.$ lit. $^{3}+6.3\left(c=2.3, \mathrm{CHCl}_{3}\right)$ (ent)-4a); IR $\mathrm{v}_{\max } / \mathrm{cm}^{-1}$ (film) 3346, 2930, 2859, 1466, 1131, 1024; ${ }^{1} \mathrm{H}$ NMR (400 MHz, $\left.\mathrm{CDCl}_{3}\right) \delta$ 5.69-5.60 (m, 2H, $\left.2 \times=\mathrm{CH}\right), 4.08-4.00(\mathrm{~m}, 2 \mathrm{H}, 2 \times \mathrm{OCH}), 2.10(\mathrm{~d}, 2 \mathrm{H}, J=4, \mathrm{OH})$, 1.60-1.20 (m, 16H, $\left.8 \times \mathrm{CH}_{2}\right), 0.88(\mathrm{t}, 6 \mathrm{H}, J=7,2 \times \mathrm{Me}) ;{ }^{13} \mathrm{C}$ NMR $\delta\left(100 \mathrm{MHz}, \mathrm{CDCl}_{3}\right)$ $133.9(2 \times=\mathrm{CH}), 72.5(2 \times \mathrm{OCH}), 37.2\left(2 \times \mathrm{CH}_{2}\right), 31.7\left(2 \times \mathrm{CH}_{2}\right), 25.1\left(2 \times \mathrm{CH}_{2}\right), 22.6(2 \times$ $\left.\mathrm{CH}_{2}\right), 14.0\left(2 \times \mathrm{CH}_{2}\right)$.

(2S,3E,5S)-1,6-Bis-(trityloxy)-hex-3-ene-2,5-diol 6

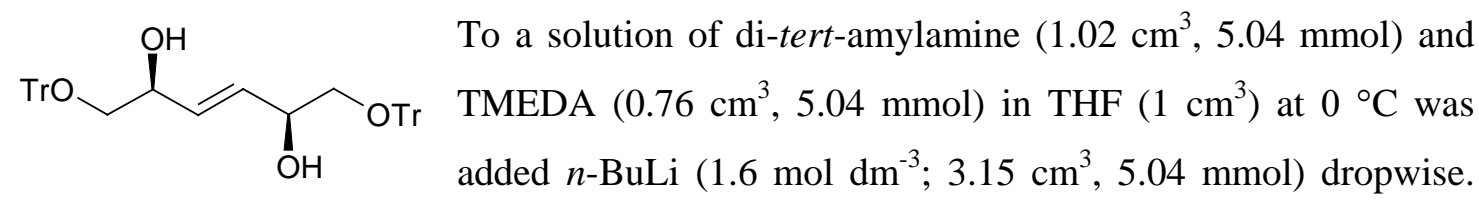

The solution was warmed to $25^{\circ} \mathrm{C}$ and stirred for $30 \mathrm{~min}$. before being cooled to $-72{ }^{\circ} \mathrm{C}$. A solution of (S)-tritylglycidyl ether (800 mg, $2.52 \mathrm{mmol})$ in THF $\left(1 \mathrm{~cm}^{3}\right)$ was added dropwise over $10 \mathrm{~min}$ and the solution was then stirred at this temperature for $40 \mathrm{~h}$. The reaction was quenched with $\mathrm{MeOH}\left(2.5 \mathrm{~cm}^{3}\right)$. The reaction mixture was separated between EtOAc (10 $\left.\mathrm{cm}^{3}\right)$ and sat. aq. $\mathrm{NH}_{4} \mathrm{Cl}\left(10 \mathrm{~cm}^{3}\right)$. The aqueous layer was washed with EtOAc $\left(10 \mathrm{~cm}^{3}\right)$ and the combined organic phases were then dried $\left(\mathrm{MgSO}_{4}\right)$, filtered and the solvent removed in vacuo. Purification of the residue by column chromatography (Florisil, 30\% EtOAc in petrol) gave enediol 6 as a white solid (409 mg, 51\%), $R_{\mathrm{f}} 0.53$ (40\% EtOAc in petrol); mp 60$61{ }^{\circ} \mathrm{C} ;[\alpha]_{\mathrm{D}}{ }^{24}+2.2\left(c=1.0, \mathrm{CHCl}_{3}\right), \mathrm{IR} \mathrm{V}_{\max } / \mathrm{cm}^{-1}(\mathrm{KBr}) 3424,3057,2921,2869,1596,1490$, 1448, 1319, 1219, 1155, 1075, 1032; ${ }^{1} \mathrm{H}$ NMR (400 MHz, $\left.\mathrm{CDCl}_{3}\right) \delta$ 7.50-7.23 (m, 30H, $2 \times$ $\left.\mathrm{CPh}_{3}\right)$, 5.73-5.71 (2H, m, $\left.2 \times=\mathrm{CH}\right), 4.31-4.28(2 \mathrm{H}, \mathrm{m}, 2 \times \mathrm{OCH}), 3.18(4 \mathrm{H}, \mathrm{ddd}, J=17,9$ and 3, $\left.2 \times \mathrm{CH}_{2} \mathrm{O}\right), 2.42(2 \mathrm{H}, \mathrm{d}, J=3,2 \times \mathrm{OH}) ;{ }^{13} \mathrm{C} \mathrm{NMR}(100 \mathrm{MHz}) \delta 143.7\left(6 \times \mathrm{ArC}_{\text {quat }}\right)$ $130.7(2 \times=\mathrm{CH}), 128.6(12 \times \mathrm{ArCH}), 127.9(12 \times \mathrm{ArCH}), 127.1(6 \times p-\mathrm{ArCH}), 86.8(2 \times$ $\left.\mathrm{OCPh}_{3}\right), 71.1(2 \times \mathrm{OCH}), 67.4\left(2 \times \mathrm{CH}_{2}\right)$; HRMS $\mathrm{m} / \mathrm{z}\left(\mathrm{M}+\mathrm{NH}_{4}^{+}\right)$Found: 650.3267 $\mathrm{C}_{44} \mathrm{H}_{44} \mathrm{O}_{4} \mathrm{~N}$ requires 650.3265 . 

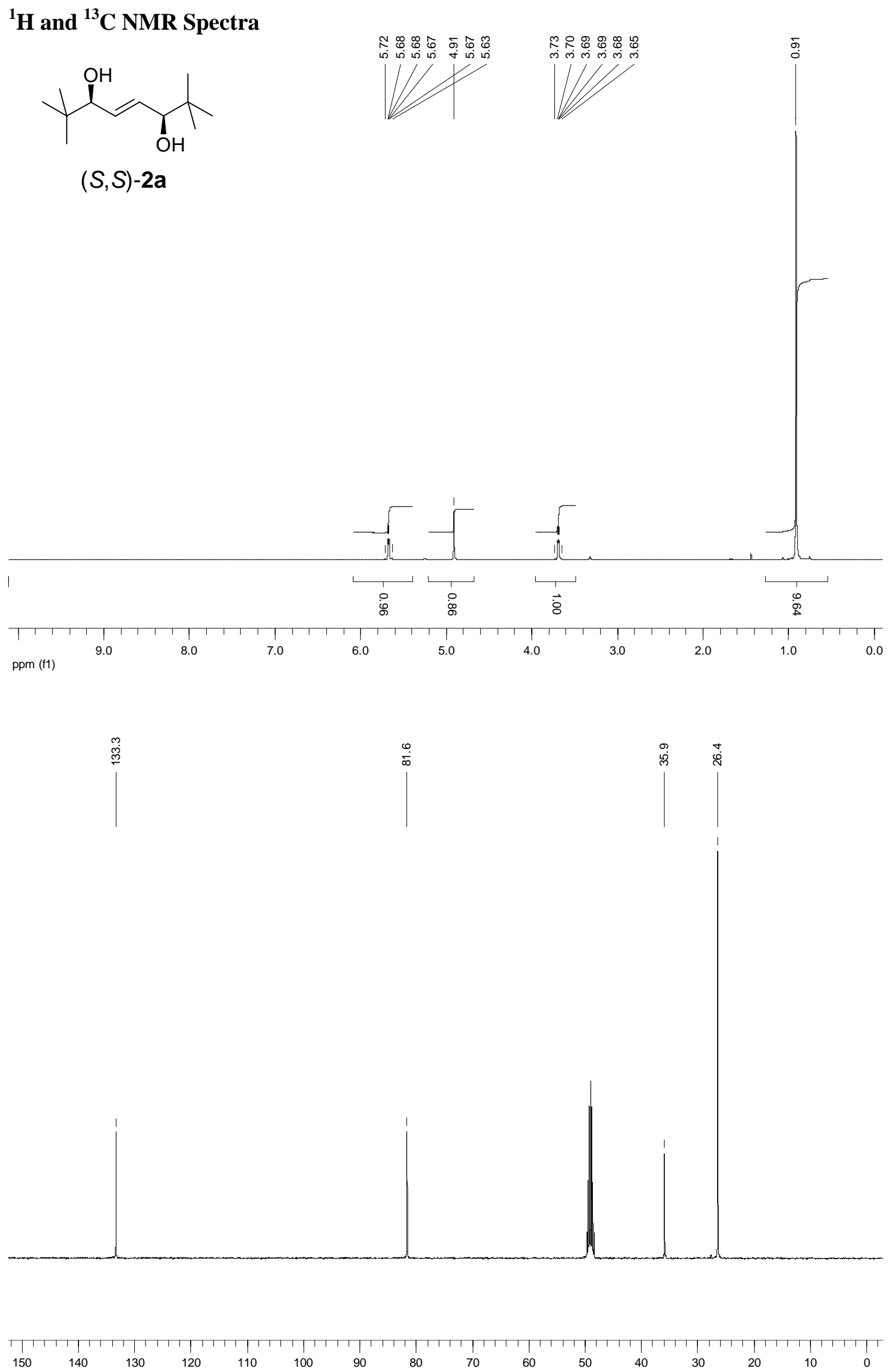


\section{S6}<smiles>OC(/C=C/[C@@H](O)C1CCCCC1)C1CCCCC1</smiles>

员品品品

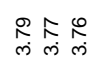

$3 a$

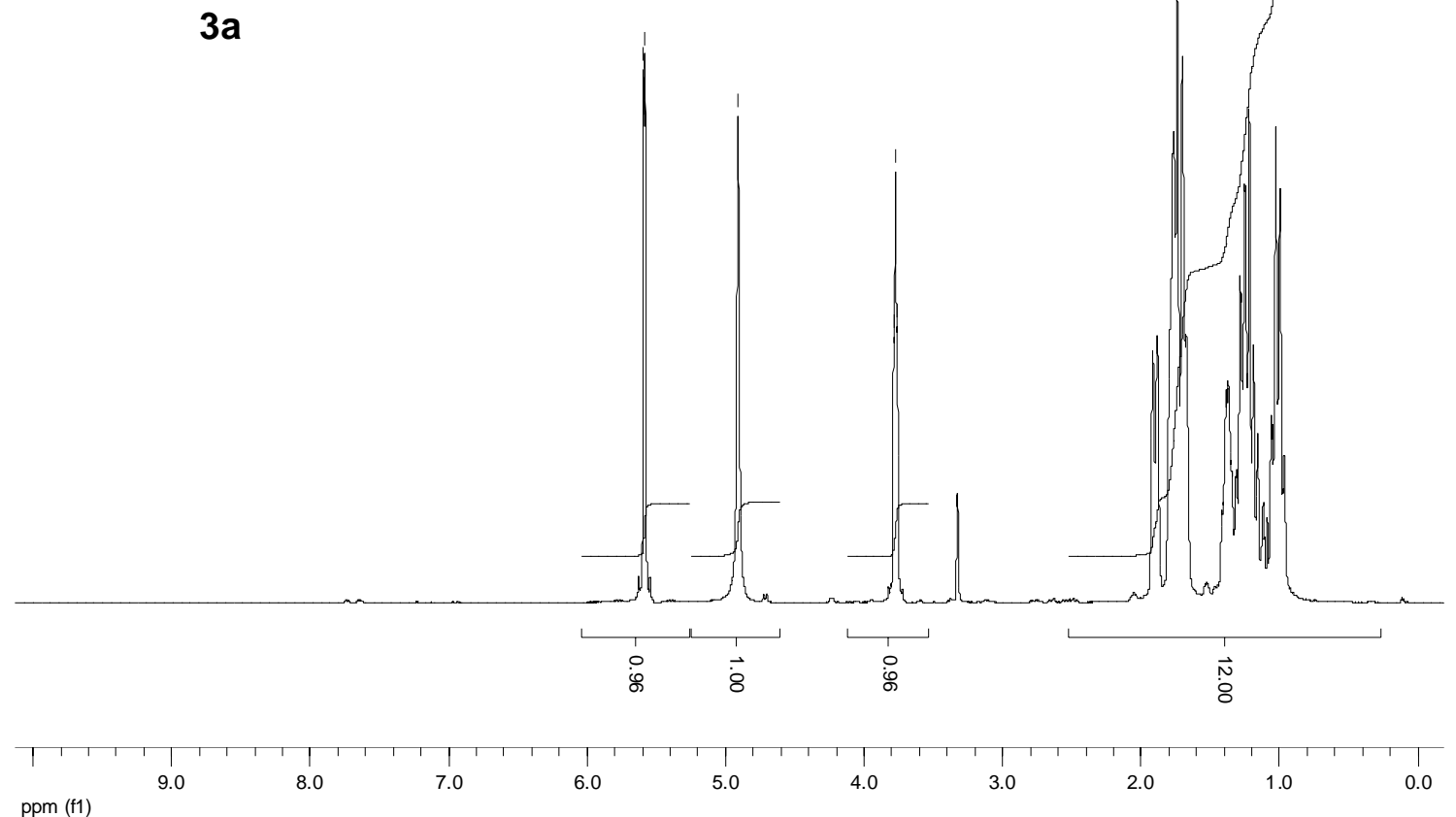

ppm (f1)

竘

$\stackrel{\infty}{\infty}$
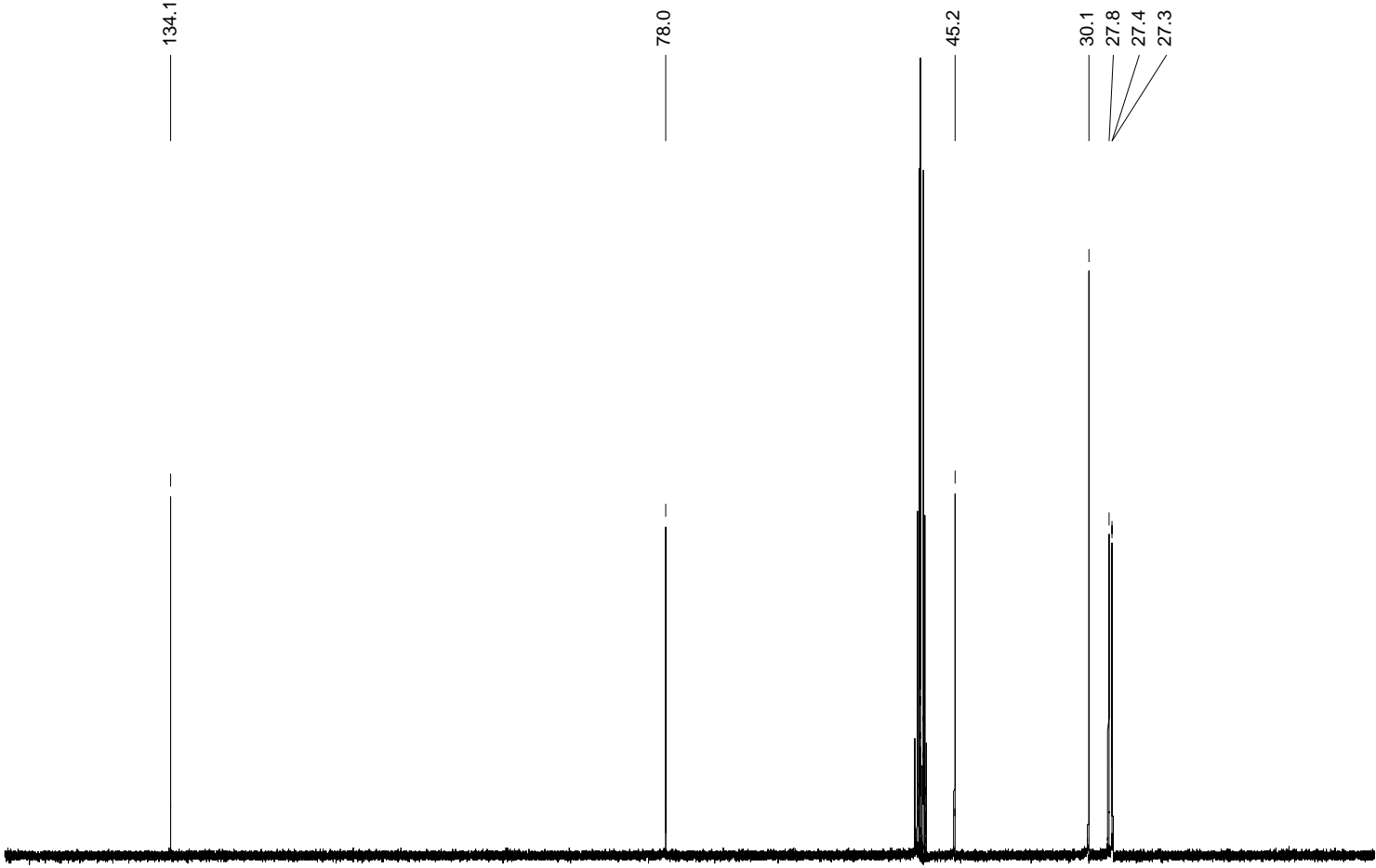

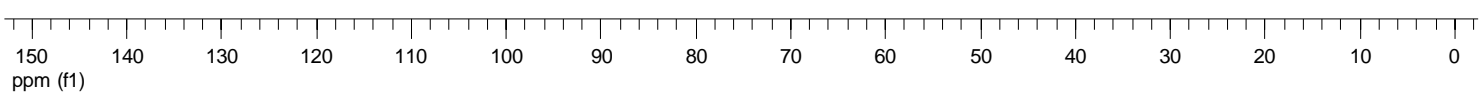




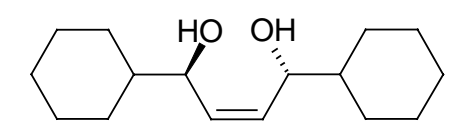

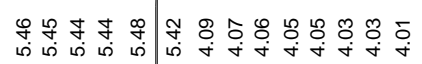

$3 b$

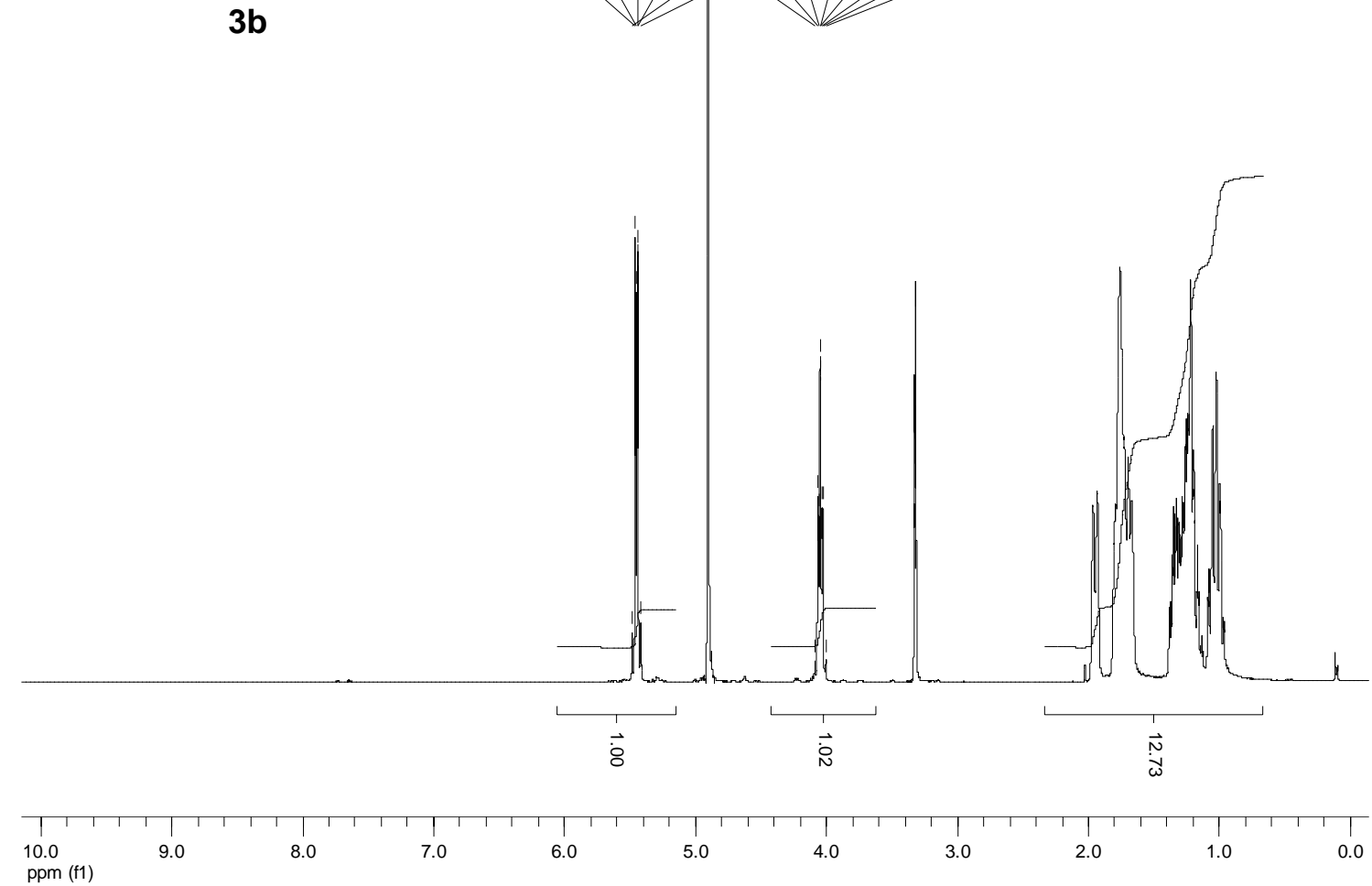

$\stackrel{\infty}{\stackrel{\infty}{m}}$
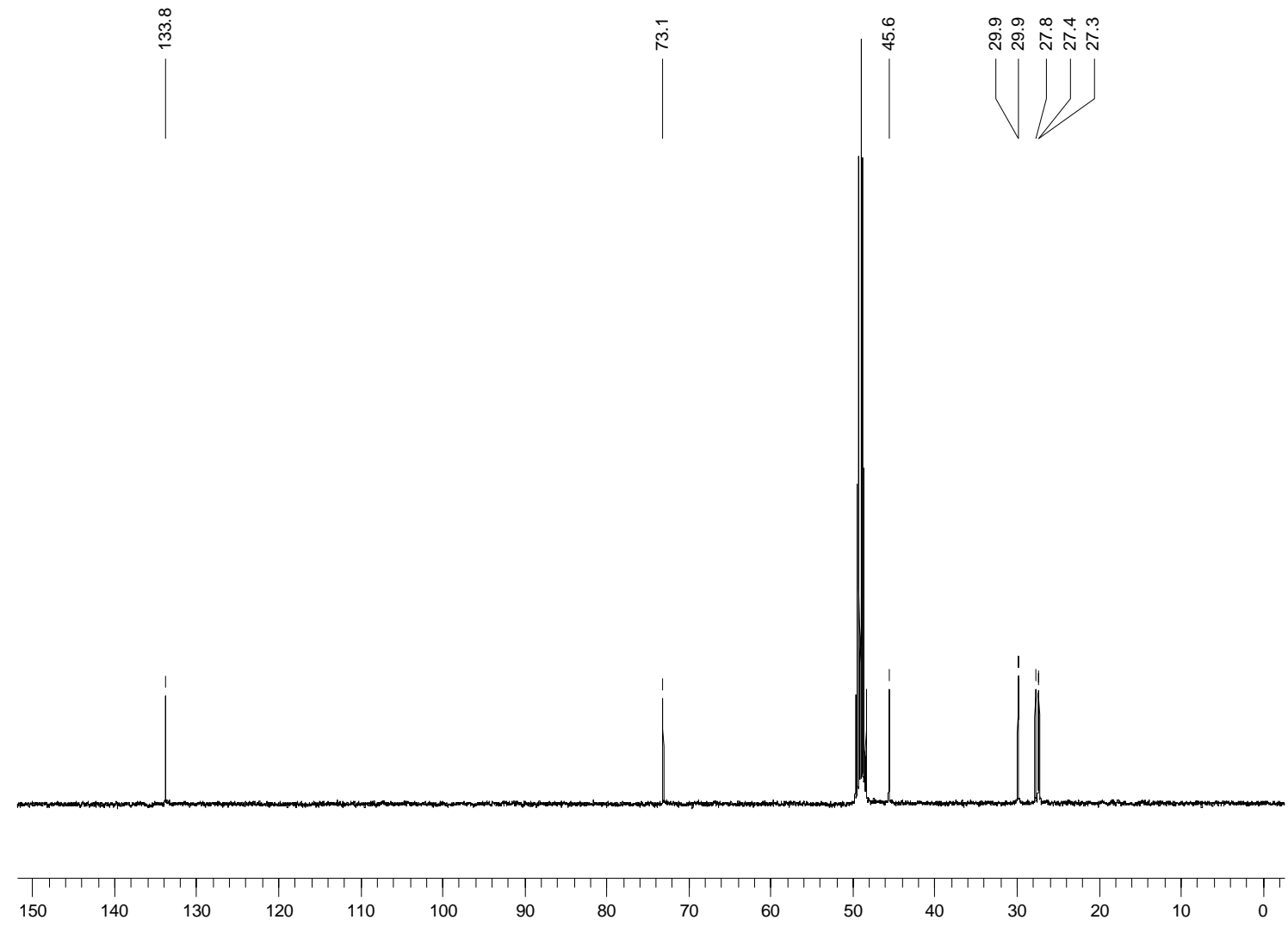

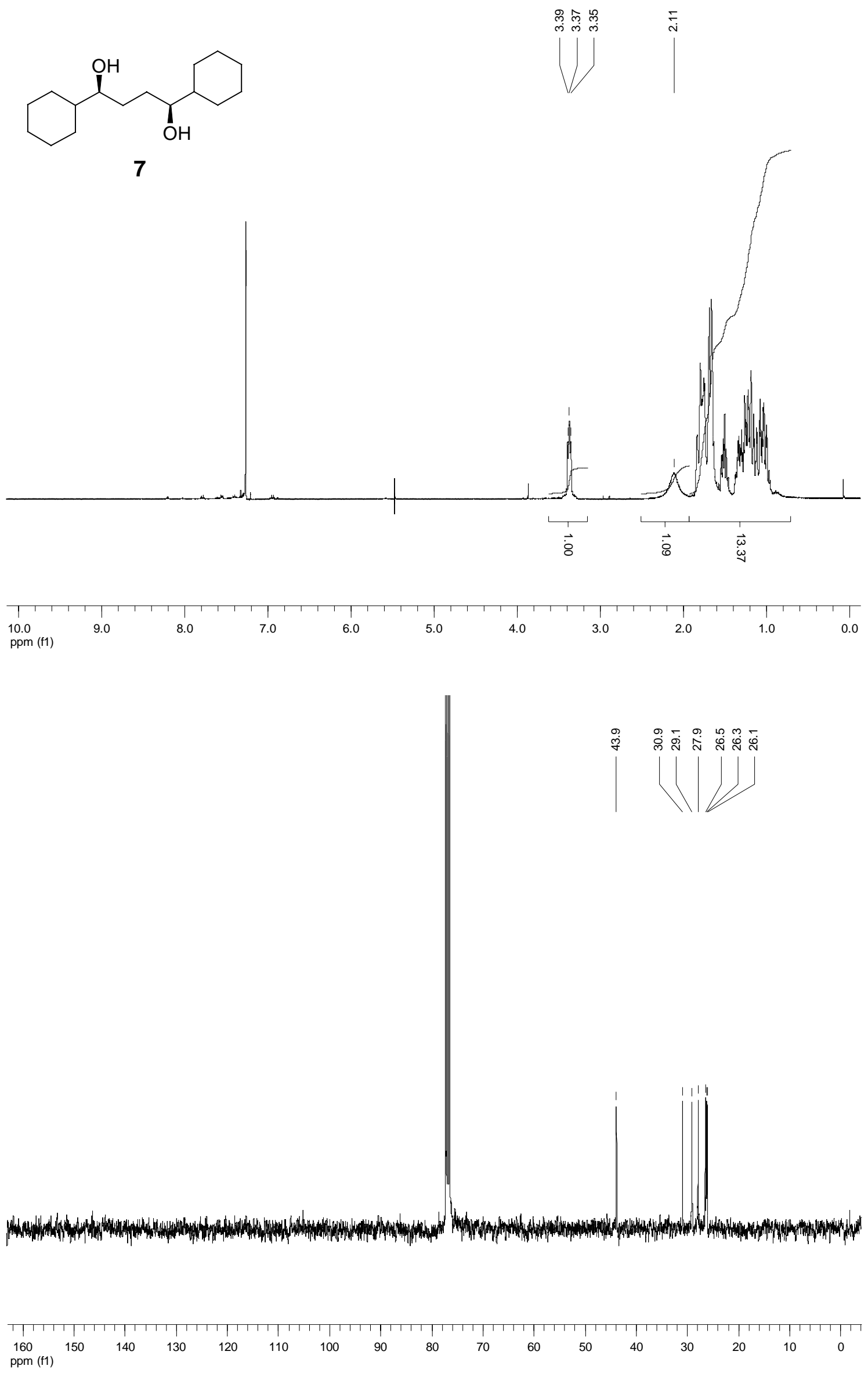


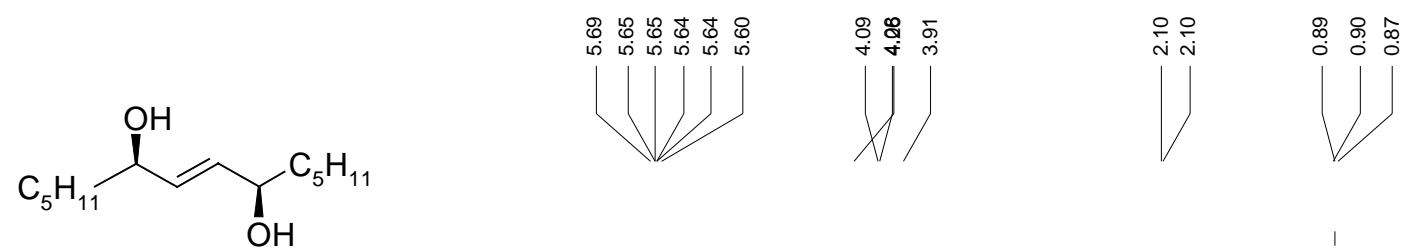

$4 a$
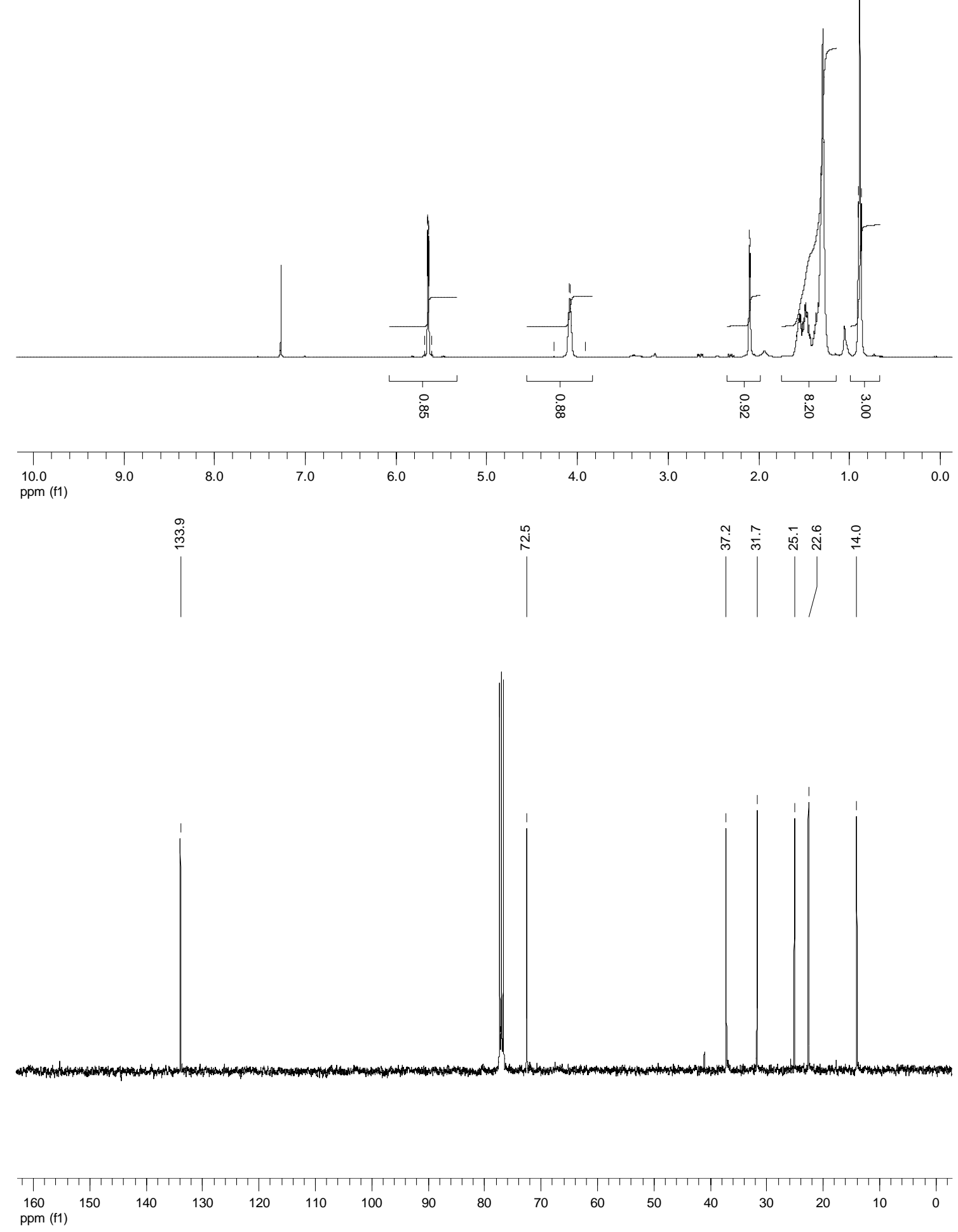


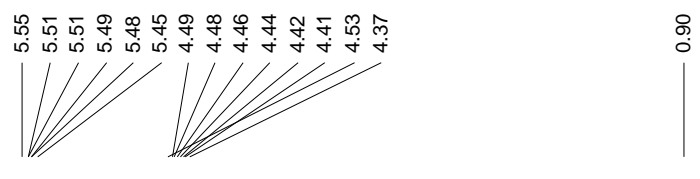

4b

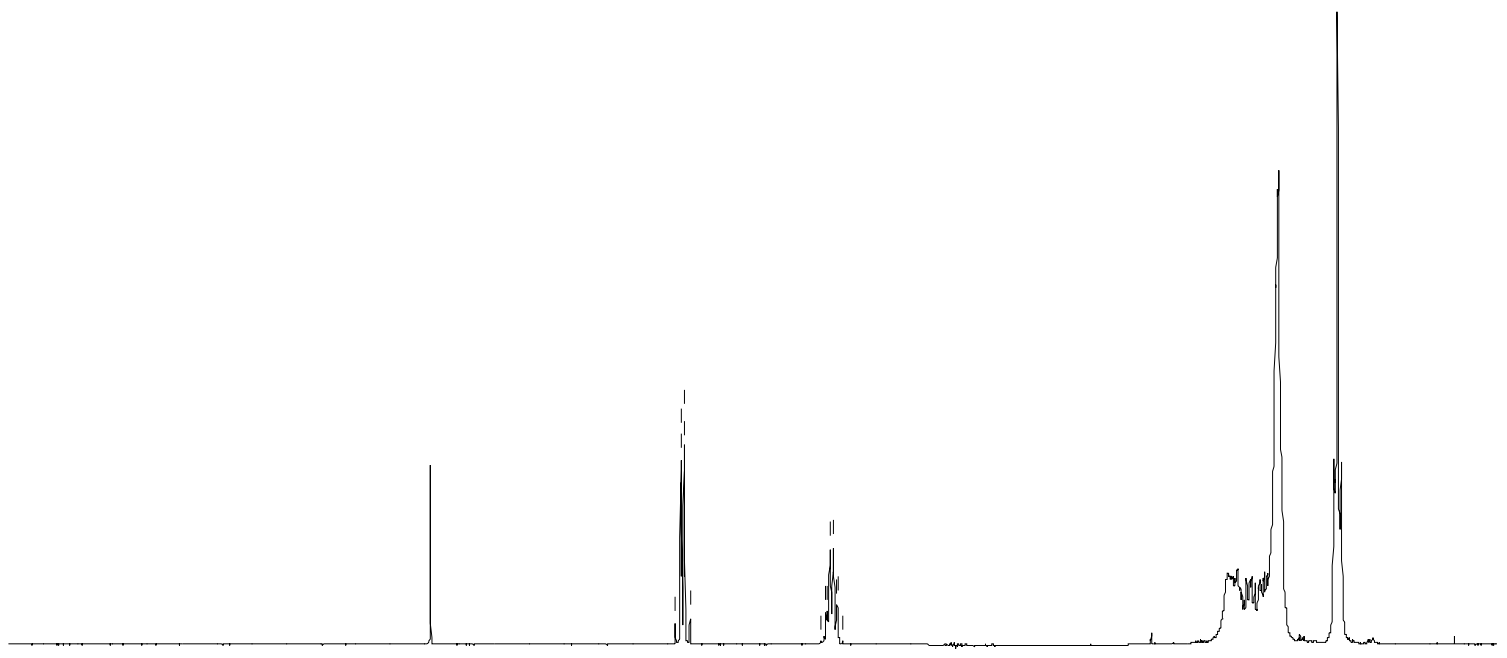

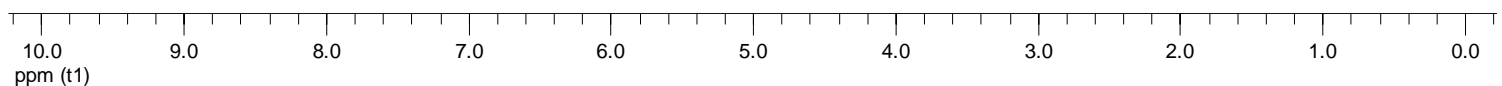

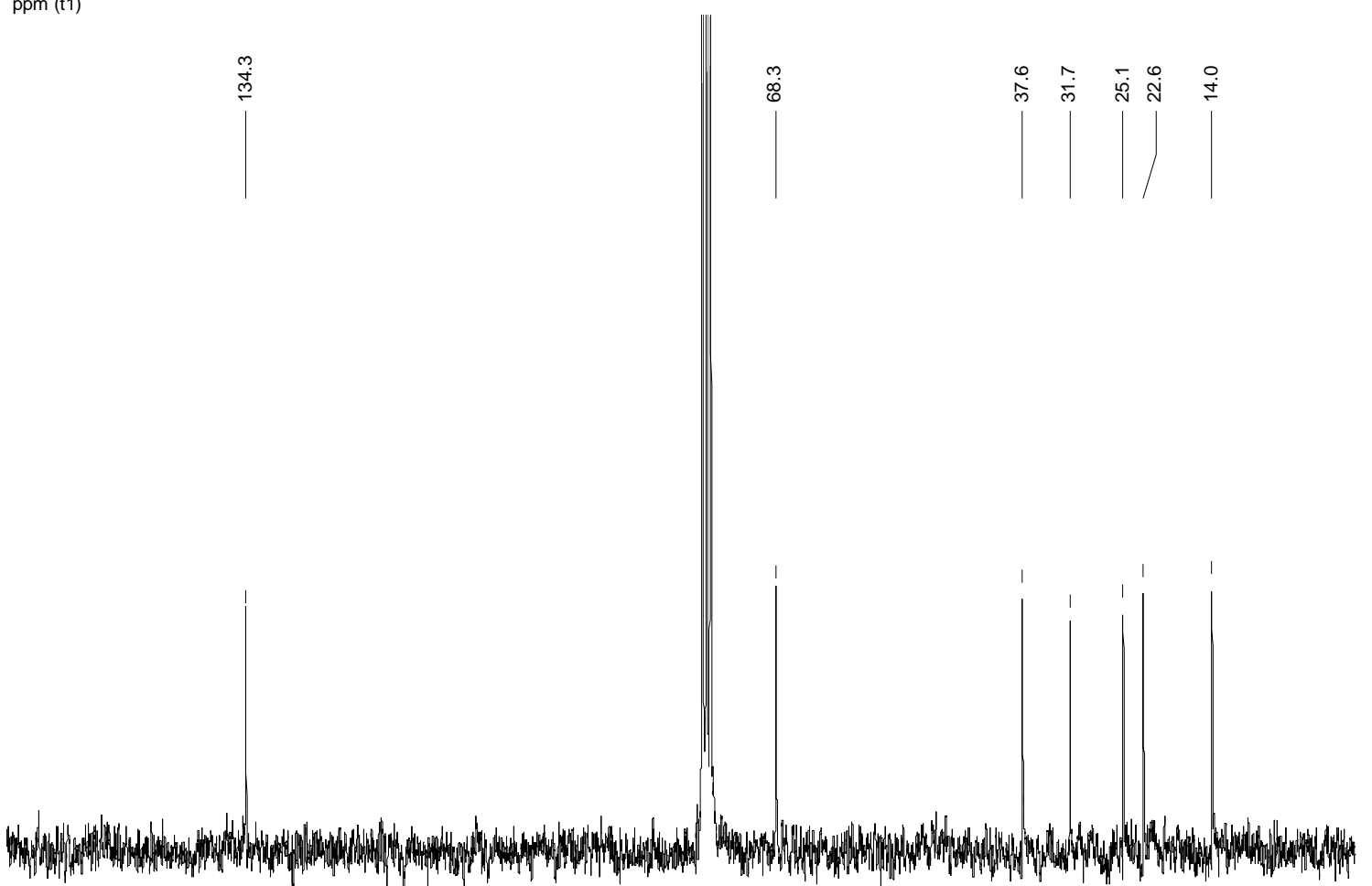

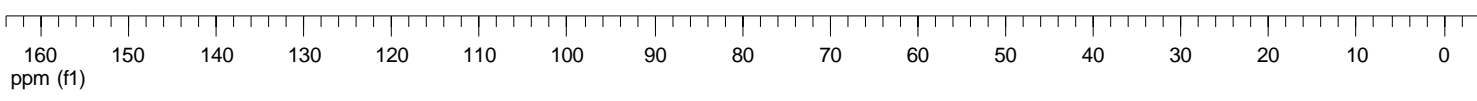



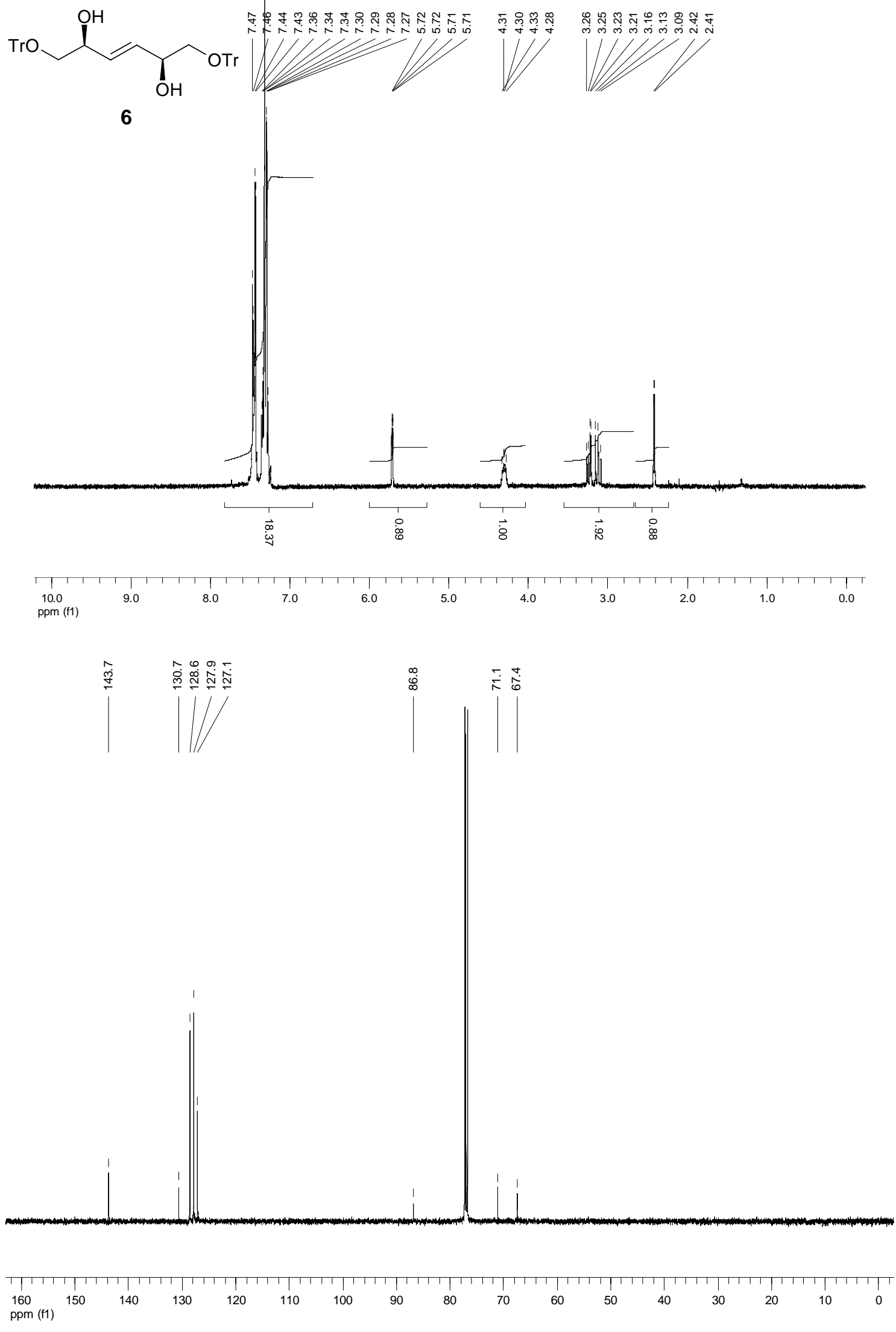


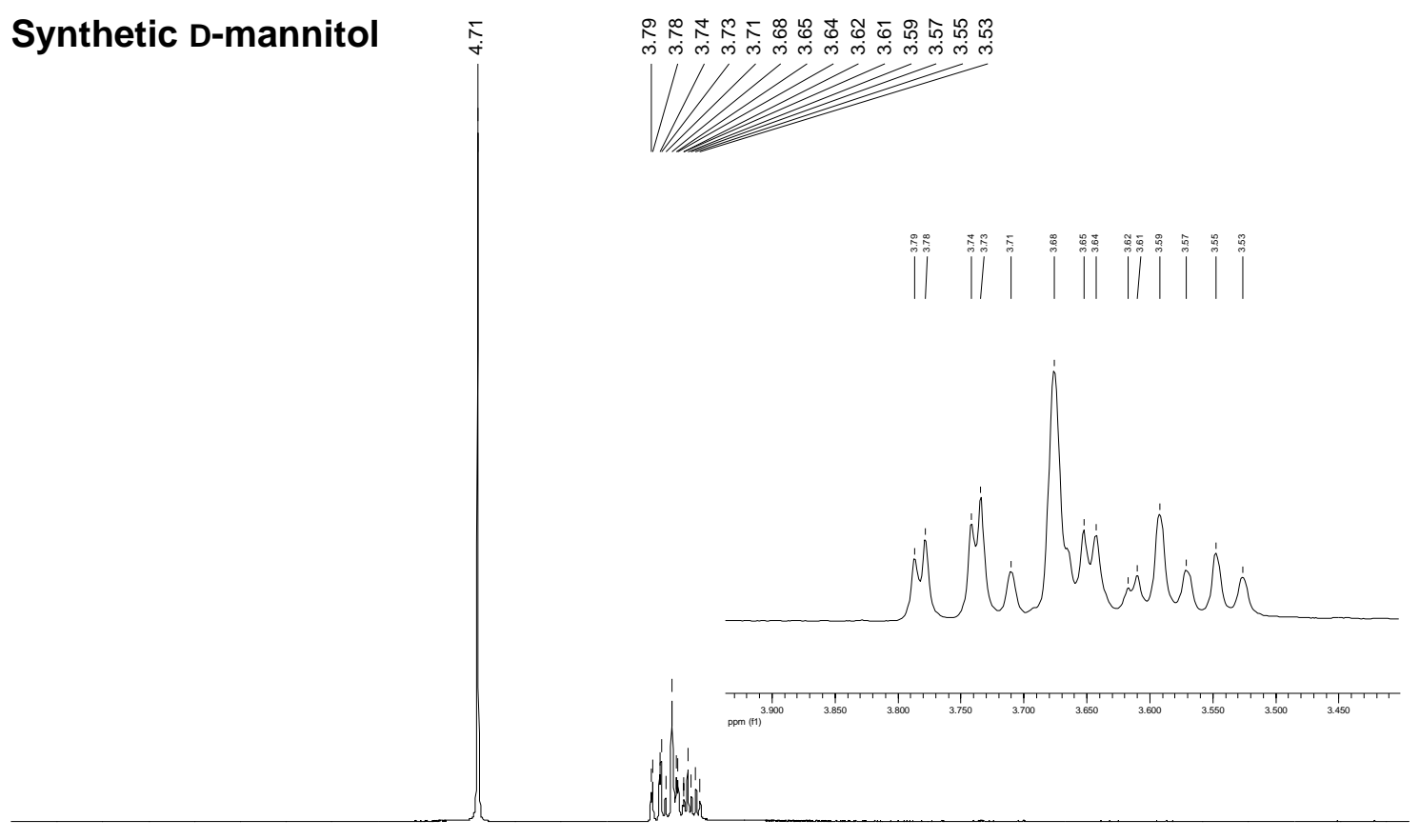

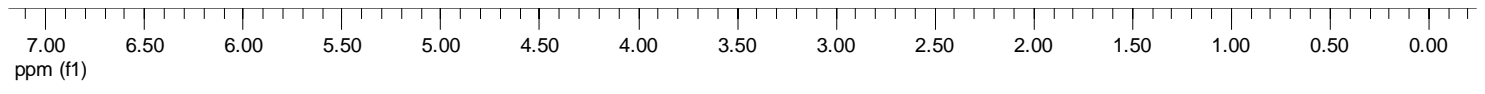

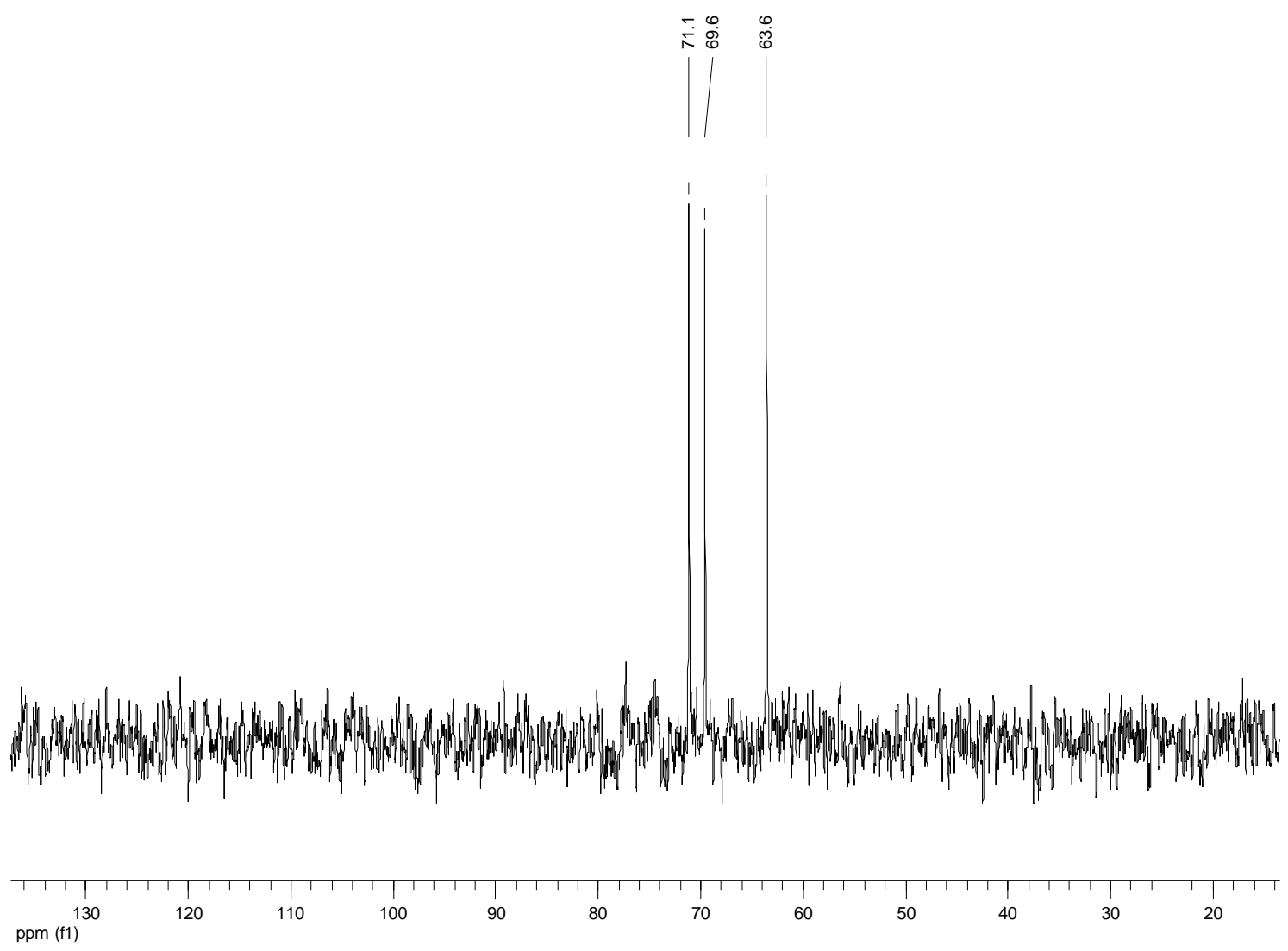




\section{Natural D-mannitol}

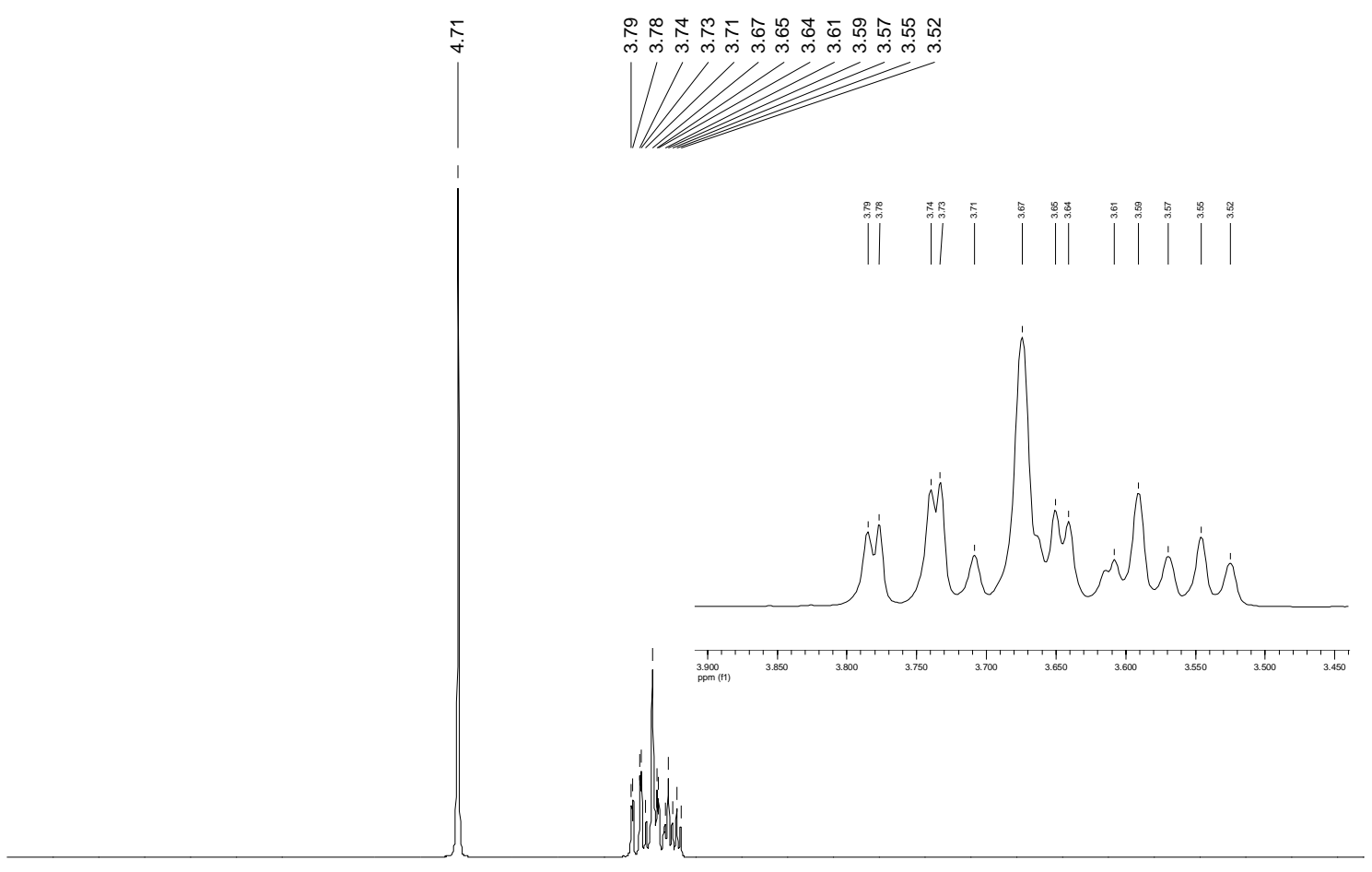

\begin{tabular}{|c|c|c|c|c|c|c|c|c|c|c|c|c|c|c|}
\hline 111 & 1 & \begin{tabular}{l|l} 
& 1
\end{tabular} & \begin{tabular}{l|l}
1 &
\end{tabular} & \begin{tabular}{l|l}
1 & 1
\end{tabular} & \begin{tabular}{l|l}
1 & 1
\end{tabular} & 1 & 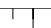 & \begin{tabular}{l|l} 
& 1
\end{tabular} & \begin{tabular}{l|l}
1 &
\end{tabular} & \begin{tabular}{l|l}
1 &
\end{tabular} & \begin{tabular}{l|l}
1 & 1
\end{tabular} & \begin{tabular}{l|l} 
& 1
\end{tabular} & \begin{tabular}{l|l}
1 & 1
\end{tabular} & 111 \\
\hline $\begin{array}{l}7.00 \\
\text { ppm (f1) }\end{array}$ & 6.50 & 6.00 & 5.50 & 5.00 & 4.50 & 4.00 & 3.50 & 3.00 & 2.50 & 2.00 & 1.50 & 1.00 & 0.50 & 0.00 \\
\hline
\end{tabular}
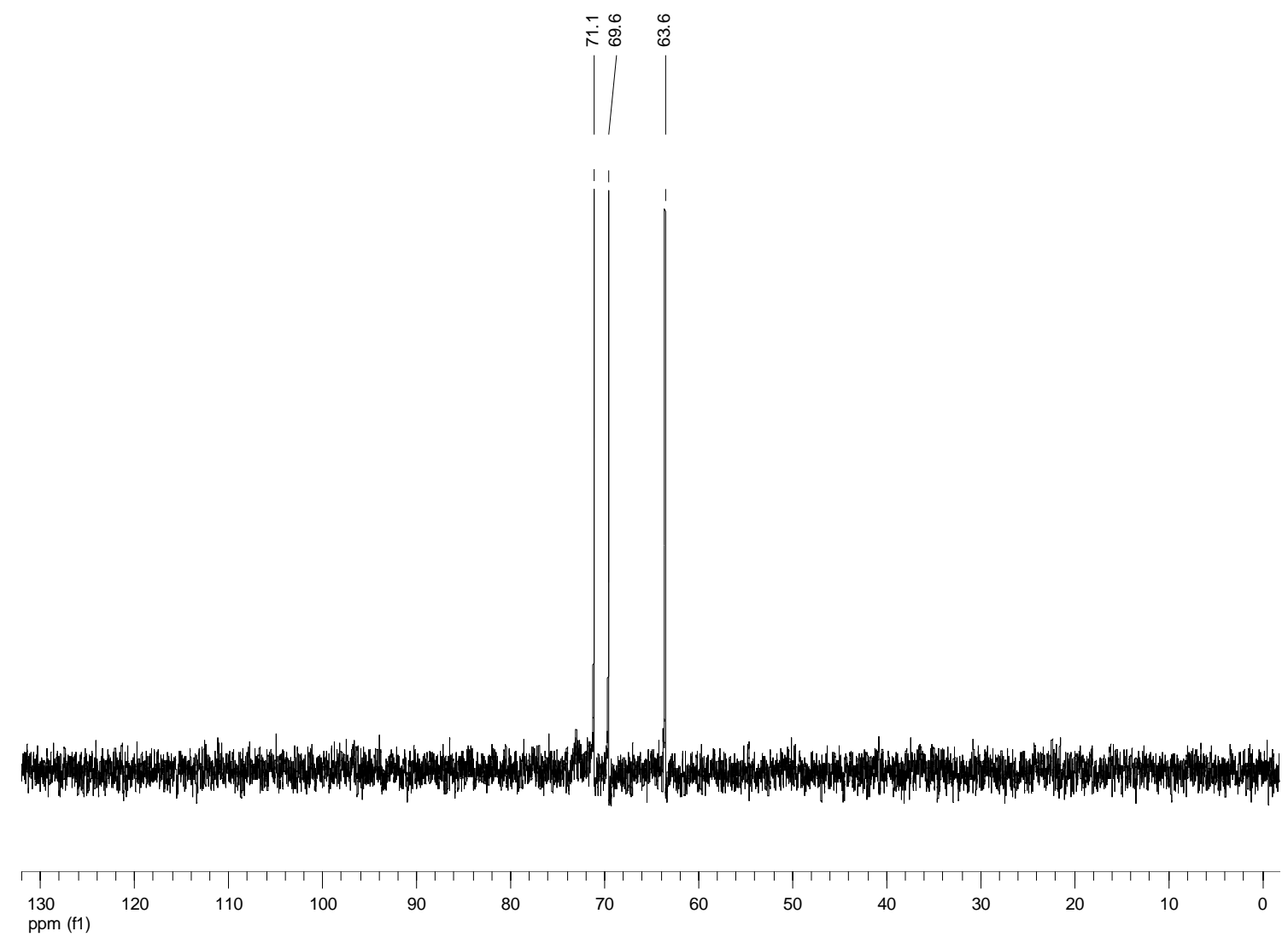


\section{Synthetic D-Iditol}
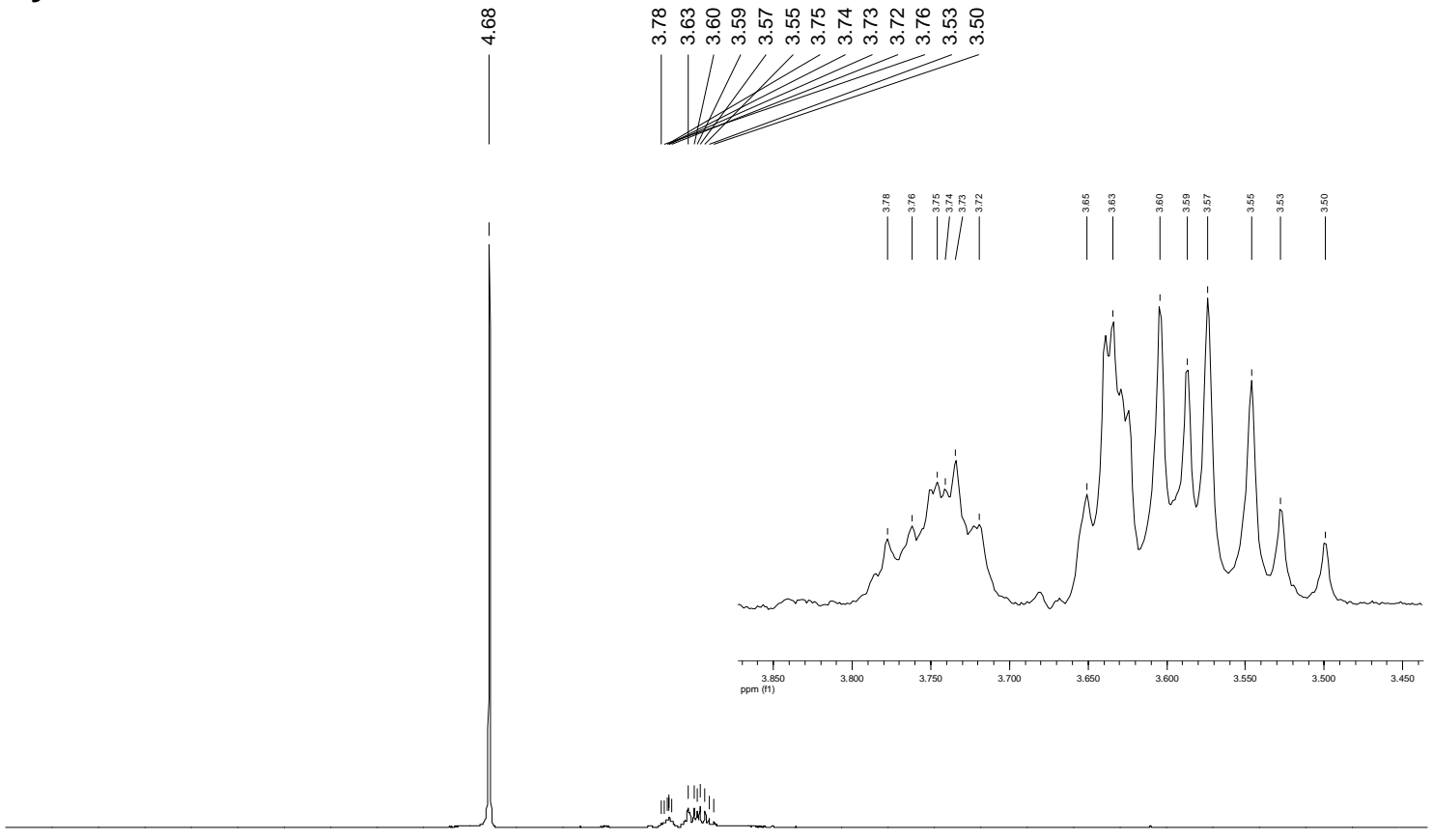

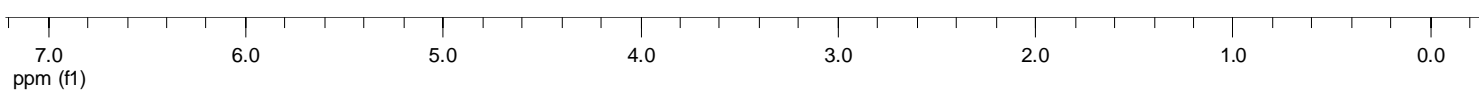

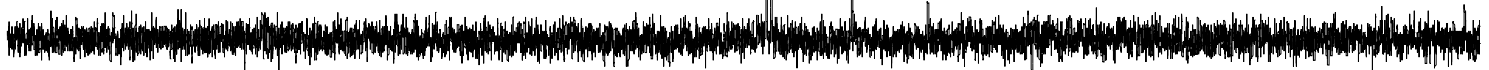

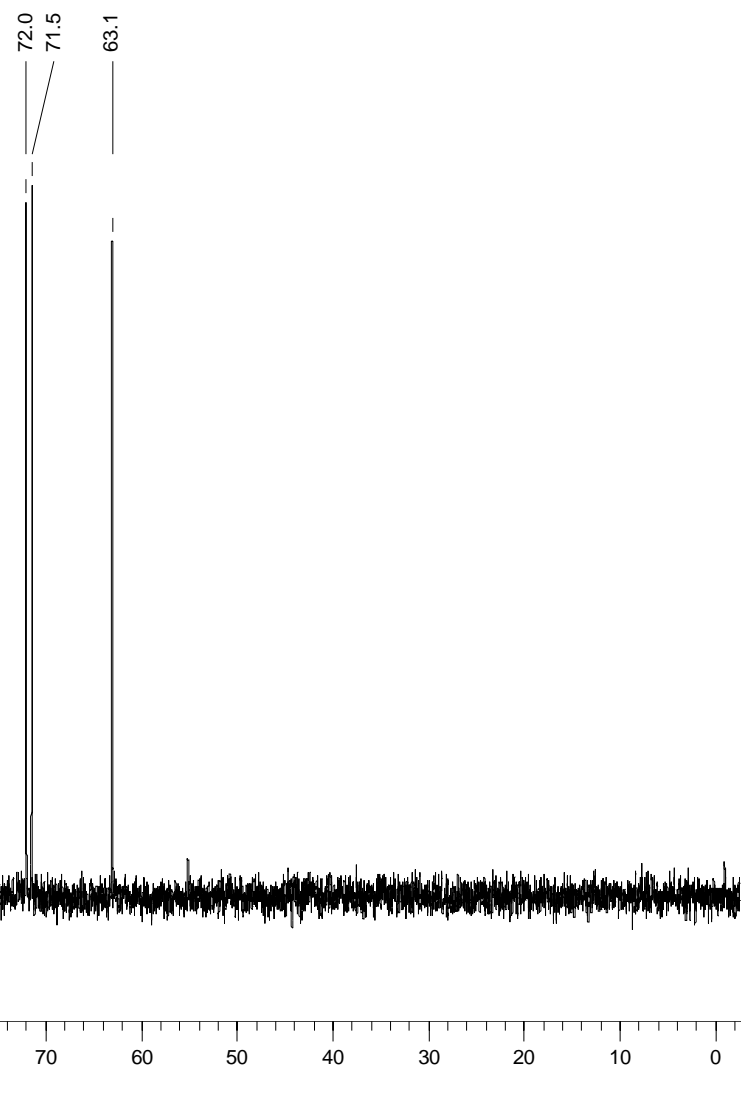

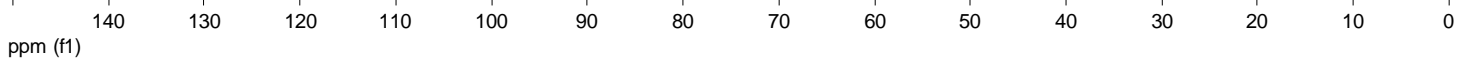




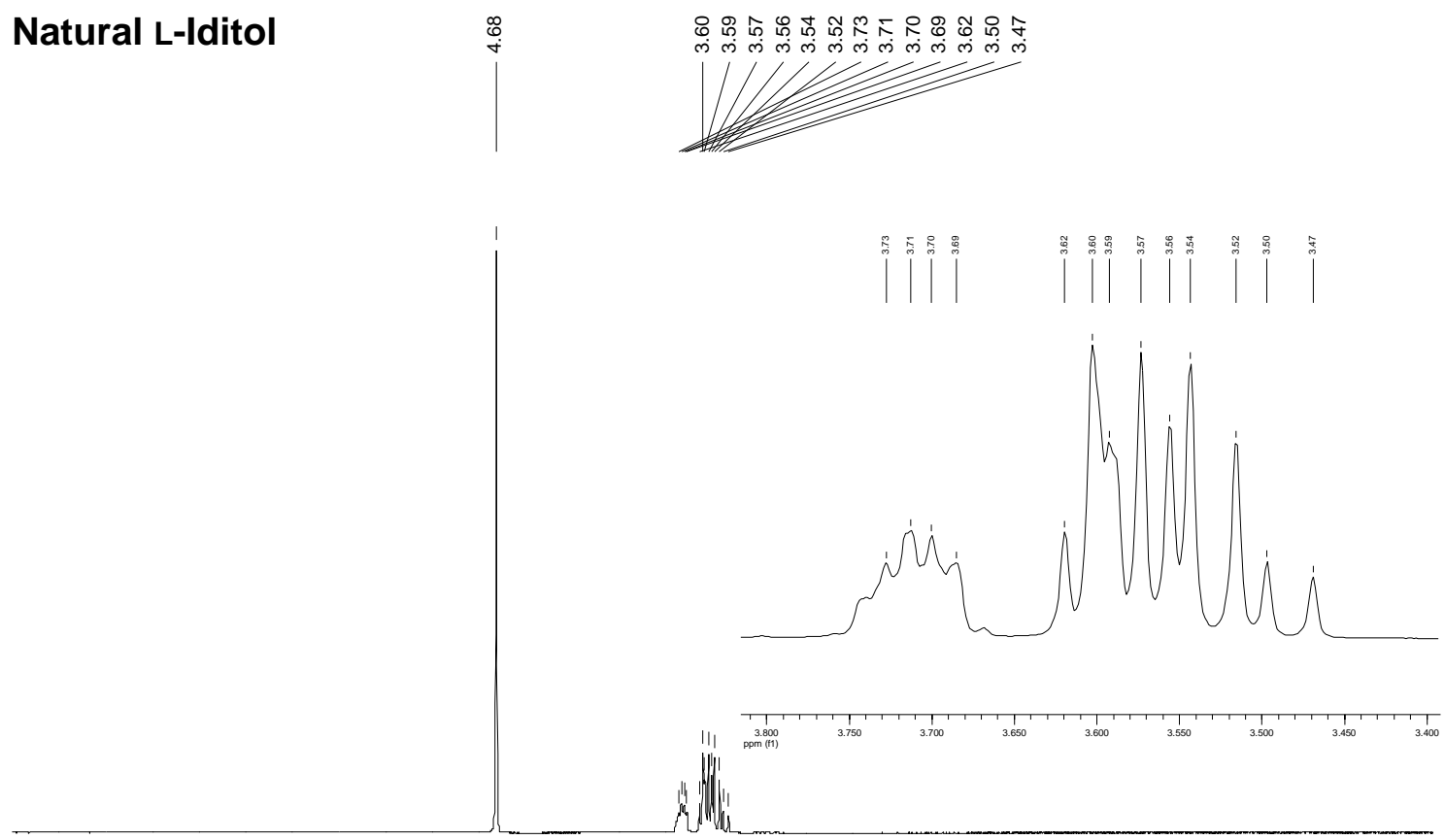

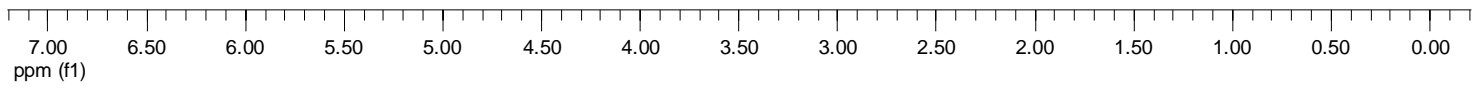

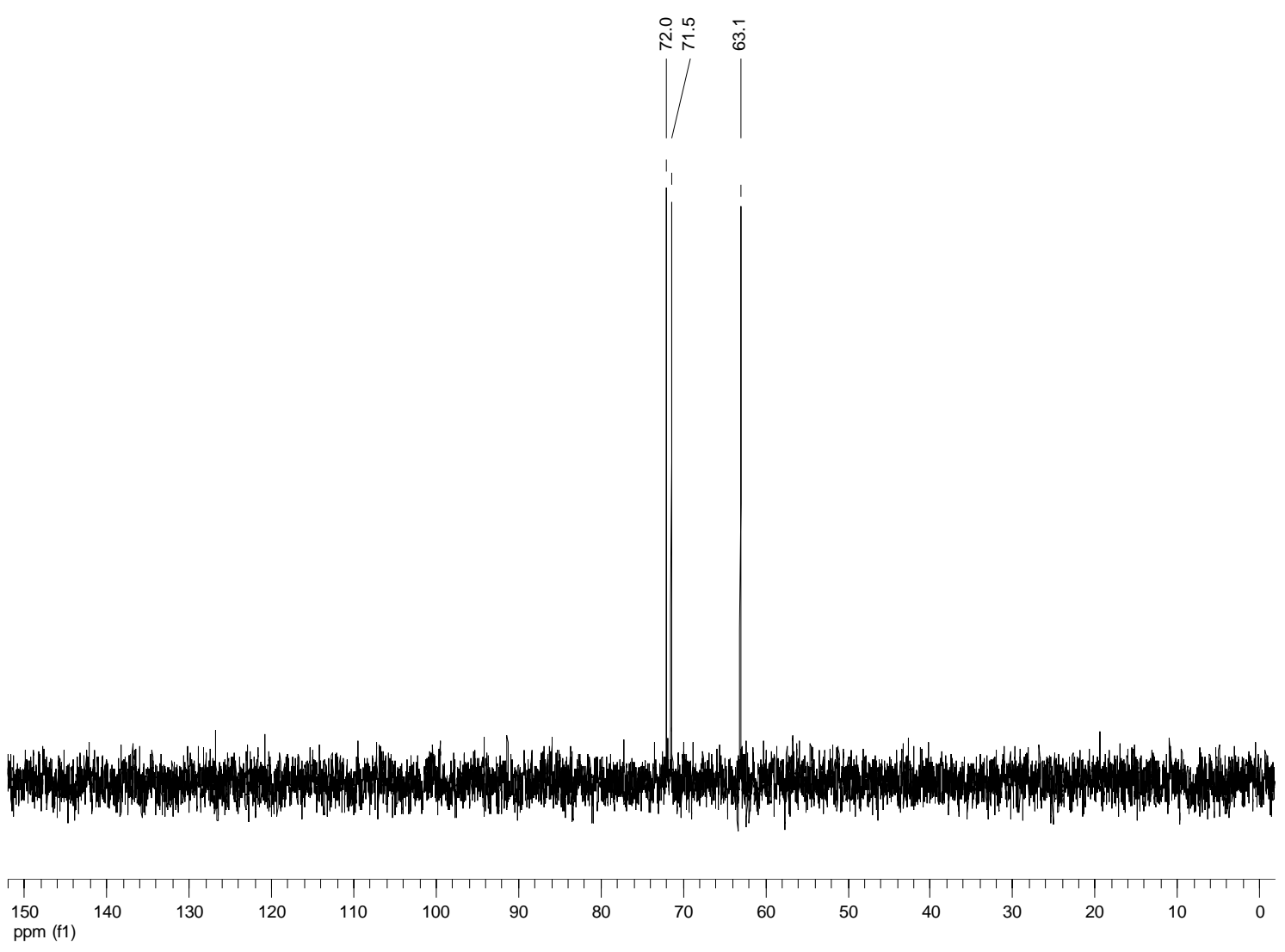


X-ray Data (orteps shown at $\mathbf{4 0 \%}$ probability)

(3S,4E, 6S)-2,2,7,7-Tetramethyloct-4-ene-3,6-diol (S,S)-2a ${ }^{4}$

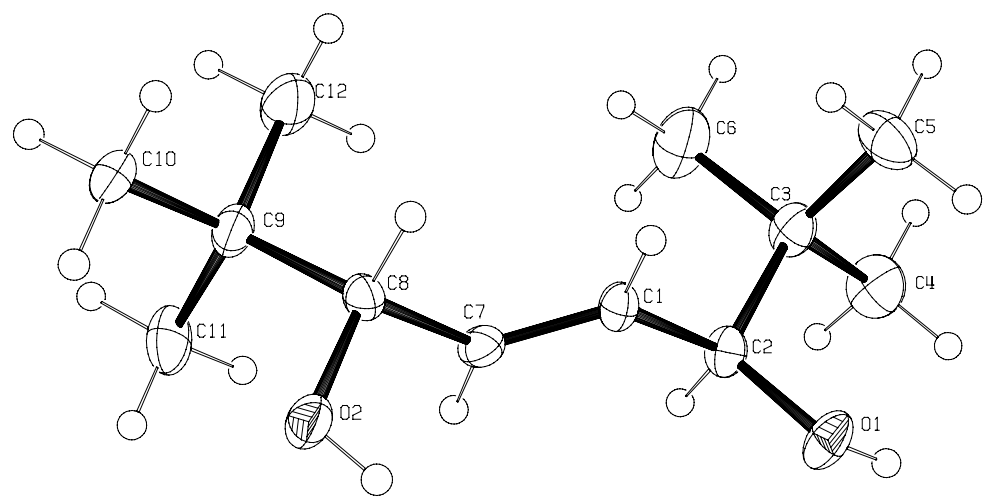

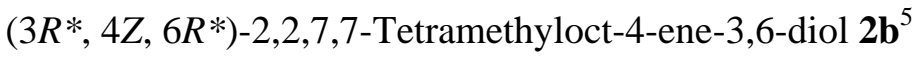

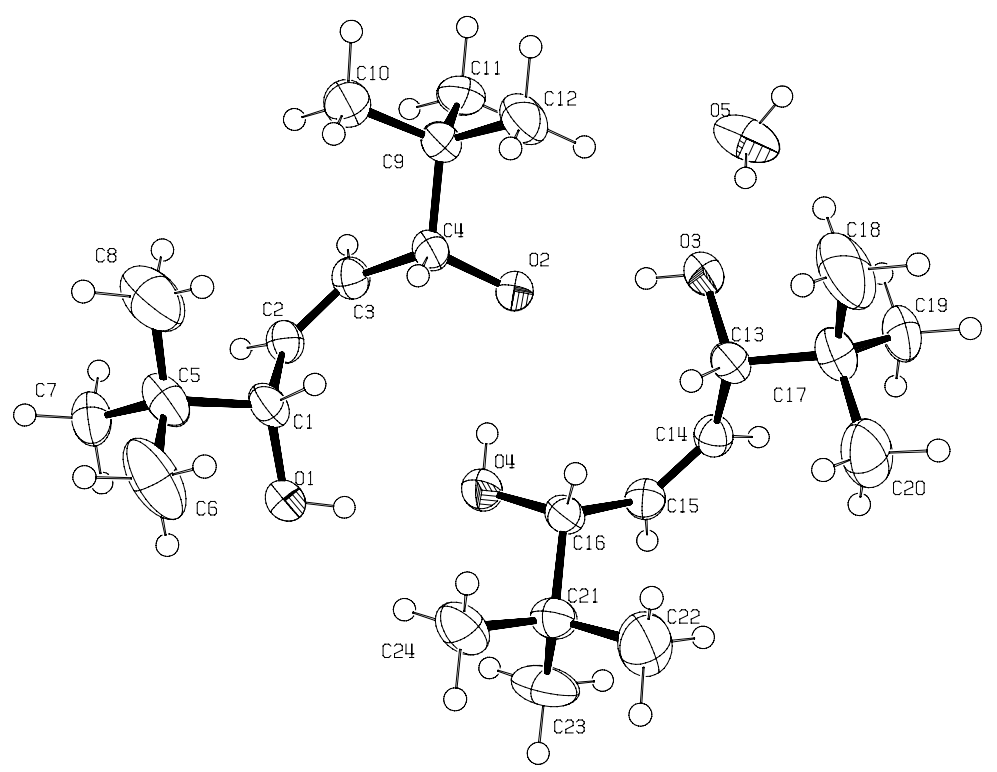

(1R,2E, 4R)-1,4-Dicyclohexylbut-2-ene-1,4-diol 3a ${ }^{6}$

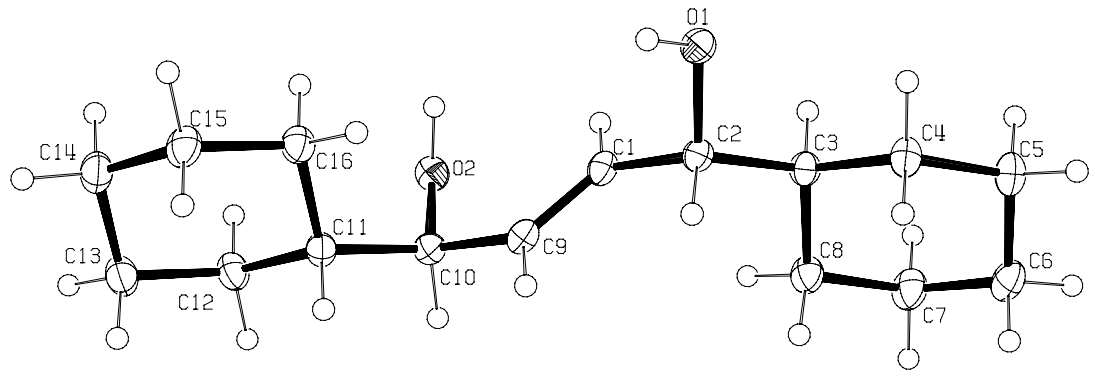




\section{References}

1. Bach, J.; Berenguer, R.; Garcia, J.; López, M.; Manzanal, J.; Vilarrasa, J. Tetrahedron 1998, 54, 14947-14962.

2. Amador, M.; Ariza, X.; Garcia, J.; Sevilla, S. Org.Lett. 2002, 4, 4511-4514.

3. Ariza, X.; Fernández, N.; Garcia, J., López, M.; Montserrat, L.; Ortiz, J. Synthesis 2004, 128-134.

4. CCDC 264354 available at http://www.ccdc.cam.ac.uk.

5. CCDC 264355 available at http://www.ccdc.cam.ac.uk.

6. CCDC 264353 available at http://www.ccdc.cam.ac.uk. 